\title{
High-Frequency Percussive Ventilation in Cystic Fibrosis Patients With Acute Respiratory Failure: A Case Series
}

\author{
Badr Jandali ${ }^{1}$, Joel D. Mermis ${ }^{1}$, Michael S. Crosser ${ }^{1}$ \\ 1. Pulmonary and Critical Care Medicine, The University of Kansas Health System, Kansas City, USA
}

Corresponding author: Badr Jandali, badrjandali@hotmail.com

\begin{abstract}
Acute respiratory failure in cystic fibrosis carries a high risk of mortality. The optimal mode of mechanical ventilation (MV) in this population is not well established. In this case series, we identified patients with cystic fibrosis who were ventilated with high-frequency percussive ventilation (HFPV) at our institution and describe their characteristics and outcomes. The use of high-frequency percussive ventilation has been sparsely described in the literature. This case series could serve as hypothesis-generating for future research.
\end{abstract}

Categories: Internal Medicine, Pulmonology

Keywords: cystic fibrosis, mechanical ventilation, high frequency percussive ventilation, acute respiratory failure, airway clearance

\section{Introduction}

Acute respiratory failure in cystic fibrosis (CF) carries a significant risk of morbidity and mortality [1]. The short and long-term outcomes are significantly worse when mechanical ventilation (MV) is needed. The optimal ventilation mode for this patient population is unknown. The benefits of chest physiotherapy and airway clearance in CF are well known [2]. This becomes of utmost importance during respiratory failure. However, once on MV, the conventional means of airway clearance are generally of limited effectiveness as patients are generally sedated and unable to complete the necessary maneuvers to facilitate effective airway clearance. Thus, on MV, accumulation of inspissated infected sputum can lead to further compromise and worsening respiratory failure. High-frequency percussive ventilation (HFPV) is a time-cycled, pressurelimited mode of percussive ventilation that delivers subphysiologic tidal volumes at rates that can exceed 500 breaths per minute. In other pulmonary diseases with airway debris, HFPV is employed as the percussive airflow facilitates secretion mobilization and clearance after endotracheal intubation takes place [3]. While this approach has not been studied in CF, the use of HFPV in thermal inhalation injuries has consistently shown less incidence of ventilator-associated pneumonia (VAP), improved oxygenation and ventilation, and possibly improved mortality [3-5].

Review began 06/07/2021 Review ended 06/24/2021 Published 07/01/2021

\section{() Copyright 2021}

Jandali et al. This is an open access article distributed under the terms of the Creative Commons Attribution License CC-BY 4.0., which permits unrestricted use, distribution, and reproduction in any medium, provided the original author and source are credited.
We reviewed the available literature and could only find a single case report of using HFPV in a cystic fibrosis pediatric patient [6]. Given poor outcomes on conventional MV and mechanistic plausibility supporting HFPV, we have been using this mode of ventilation at our institution with our adult CF population. We aim to describe our experience with this case series.

\section{Materials And Methods}

The institutional review board approval for data collection was obtained before any data gathering. The medical record Epic (Epic Systems, Verona, USA) was queried using HERON (Healthcare Enterprise Repository for Ontological Narration) software (University of Kansas Medical Center, Kansas City, USA) with appropriate ICD-9/ICD-10 codes to identify patients who had cystic fibrosis and required mechanical ventilation using high-frequency percussive ventilation. A list of 12 patient encounters was identified. This was cross-referenced and confirmed with our departmental CF registry. Patients' charts were individually checked to confirm cystic fibrosis diagnosis (defined by sweat chloride $>60 \mathrm{mmol} / \mathrm{L}$ or two known CFcausing mutations) and identify those who were ventilated using HFPV. One patient was excluded due to lung transplant status and one patient had two encounters that are reported separately. The identified patients were hospitalized between April 2010 to May 2019 and data was collected in September 2020. Baseline forced expiratory volume in the first second (FEV1) was defined as the most recent FEV1 measured at an office visit prior to admission in a stable condition and post-hospital FEV1 was defined as the FEV1 measured at the first office visit post-hospitalization.

\section{Results}

Eleven patient encounters (four females) with CF and acute respiratory failure treated with HFPV were analyzed. Six patients survived to discharge and clinic follow-up. The median age was $30(20-40)$ years. The 


\section{Cureus}

median hospital length of stay (LOS) was 28 (15-37) days while the ICU median LOS was 9 (4-24) days. The median time spent on total MV was 4 (3-12) days and on HFPV 4 (2-11) days. The median baseline FEV1 prior to admission was 1.04L (0.73-1.62 L), 29\% (19-45\%) of predicted (Table 1).

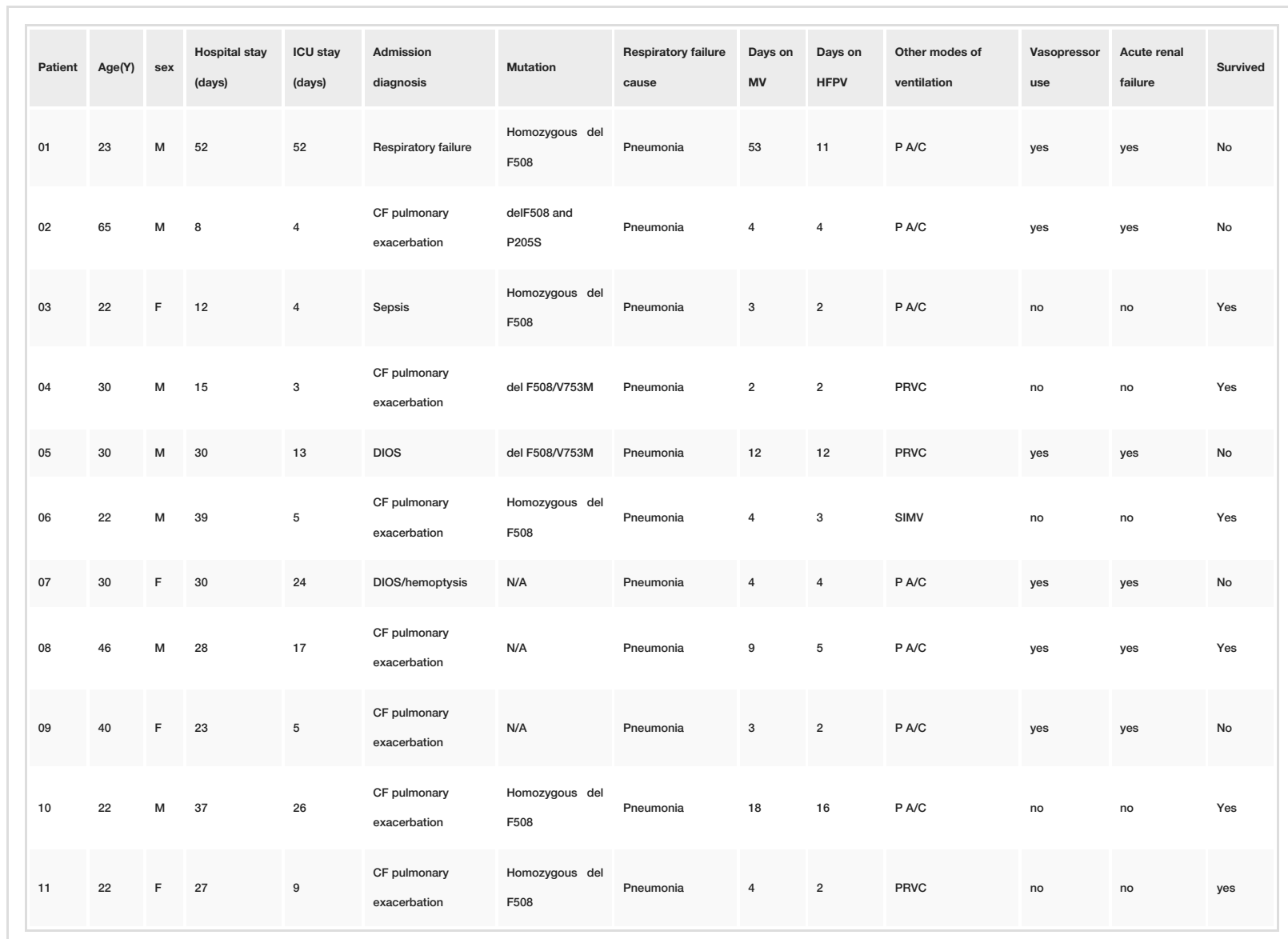

\section{TABLE 1: Patients' characteristics}

CF: cystic fibrosis; MV: mechanical ventilation; HFPV: high-frequency percussive ventilation; DIOS: distal intestinal obstructive syndrome; P A/C: pressure assist control; PRVC: pressure regulated volume assist; SIMV: synchronized intermittent mandatory ventilation

The six patients who survived to hospital discharge had a median age of 22 (22-34) years. Their hospital median LOS was 27.5 (14.25-37.5) days and median ICU LOS was 7 (3.75-19.25) days. They spent a median of 4 (3.75-11.25) days on total MV. Of those, a median of 2.5 (2-7.75) days were spent on HFPV (Figure 1).

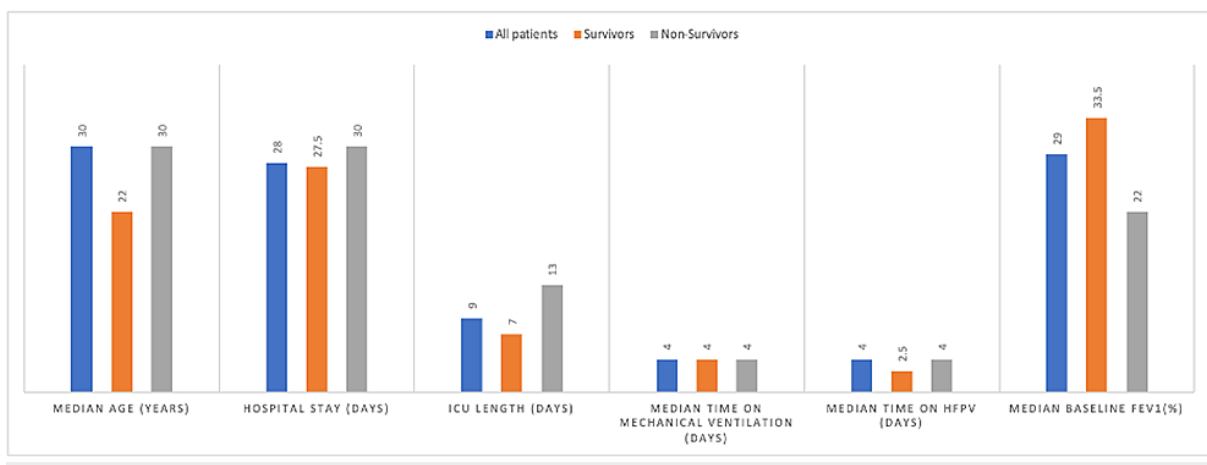

FIGURE 1: Comparison of survivors and non-survivors

Their median prior-to-admission baseline FEV1 was $1.41 \mathrm{~L}$ (33.5\% of predicted) with a median follow-up FEV1 of $1.33 \mathrm{~L}$ (32\% of predicted) (Figure 2). 


\section{Cureus}

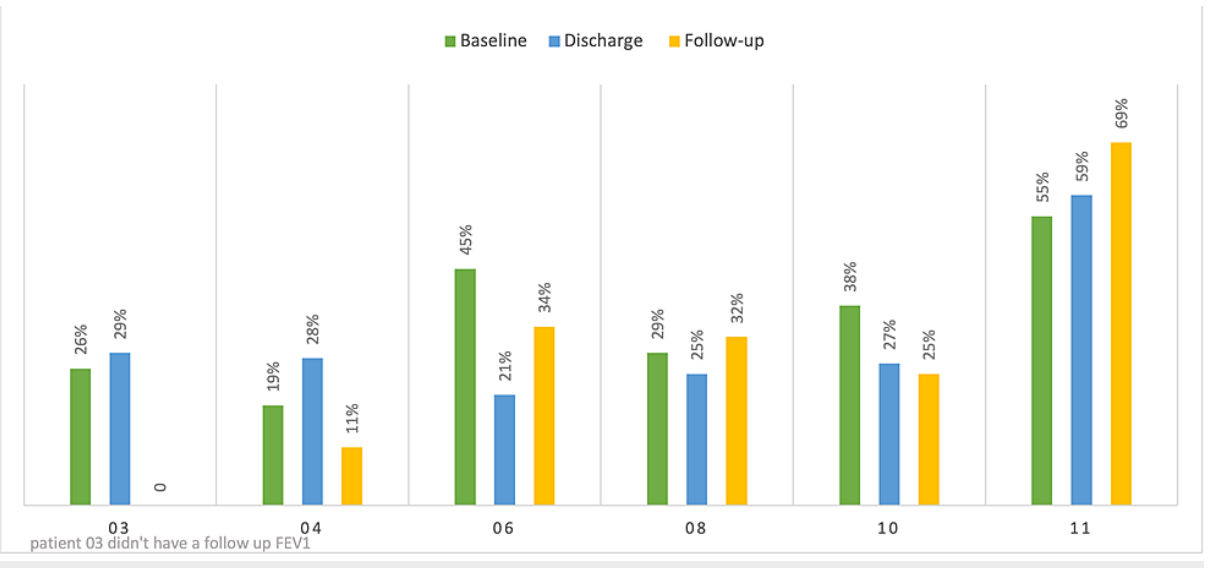

FIGURE 2: FEV1 trends of the patients who survived

Patient 3 went to transplant shortly post-discharge and had no follow-up FEV1 on our records.

FEV1: forced expiratory volume in the first second.

All the patients in this cohort had documented improved airway clearance by the ICU team after implementing HFPV without documented complications. However, this finding was limited to subjective observation and was not quantified by a standard method.

\section{Discussion}

High-frequency percussive ventilation is a subtype of high-frequency ventilation. It is a time-cycled and pressure-limited ventilator that delivers small tidal volumes using inspiratory and expiratory oscillations. The ventilator is called Volumetric Diffusive Respiration Unit (VDR-4) and comes with a Phasitron (Percussionnaire, Sandpoint, USA). This is an inspiratory and expiratory valve that is driven by a highpressure and high-frequency gas supply superimposed on conventional pressure-controlled cycles [7].

There are multiple mechanisms that contribute to gas exchange during HFPV. Aside from the direct bulk flow that is observed with conventional ventilation, HFPV creates an asymmetric velocity profile with a laminar flow pattern in which gas in the center of the airway advances inward and gas outside the center flows in a retrospect fashion [8]. This flow pattern along with the continuous pulsatory percussive waves, aid the lysis and mobilization of inspissated viscous secretions in cystic fibrosis patients [9].

While it is possible to use conventional mechanical ventilation with intermittent intrapulmonary percussive pressure, HFPV offers the potential advantage of continuous secretions mobilization and minute-to-minute airway clearance for $\mathrm{CF}$ patients. This is especially beneficial when cough reflexes are suppressed with sedation and when mucous production is at a peak with active infection.

In this case series, we report the outcomes of 10 cystic fibrosis patients ( 11 hospital encounters) in whom HFPV was used during invasive MV. While small, this CF cohort does appear to represent the adult CF ICU patient in terms of initial diagnosis, the severity of illness, and age. All of the patients were admitted with primary respiratory and CF-related diagnoses. All of the MV indications were directly related to primary respiratory failure. As expected, the median baseline FEV1 of $29 \%$ does suggest advanced $\mathrm{CF}$ lung disease and poor lung function at baseline.

The in-hospital mortality in this case series is five out of 11 patients, which is consistent with a recent national cross-sectional study where Siuba et al found a mortality rate of $44.5 \%$ for cystic fibrosis patients requiring MV [1]. The modes of ventilation were not defined in that study, but our numbers would at least suggest mortality outcomes consistent with the national trend. Respiratory therapy documentation and physician progress notes all noted significant improvement in airway clearance (secretion mobilization via endotracheal tube) after HFPV implementation, but this finding was not uniformly quantified by a standard method and could be prone to bias. However, it is consistent with the authors' experience and with non-CF trials on HFPV, especially in thermal inhalation injuries. In addition, the HFPV offers an added advantage of using lower peak airway pressures and decreases the risk of dynamic hyperinflation by using smaller tidal volumes. None of the patients in our cohort suffered from pneumothorax or ventilator-related complications.

\section{Conclusions}

High-frequency percussive ventilation appears to be safe, has the potential to augment airway clearance, 
and is at least as effective as conventional ventilation for cystic fibrosis patients who require invasive MV. Further trials would be needed to demonstrate superiority versus the current standard of care.

\section{Additional Information \\ Disclosures}

Human subjects: Consent was obtained or waived by all participants in this study. Animal subjects: All authors have confirmed that this study did not involve animal subjects or tissue. Conflicts of interest: In compliance with the ICMJE uniform disclosure form, all authors declare the following: Payment/services info: All authors have declared that no financial support was received from any organization for the submitted work. Financial relationships: All authors have declared that they have no financial relationships at present or within the previous three years with any organizations that might have an interest in the submitted work. Other relationships: All authors have declared that there are no other relationships or activities that could appear to have influenced the submitted work.

\section{References}

1. Siuba M, Attaway A, Zein J, et al.: Mortality in adults with cystic fibrosis requiring mechanical ventilation. Cross-sectional analysis of nationwide events. Ann Am Thorac Soc. 2019, 16:1017-23.

10.1513/AnnalsATS.201804-2680C

2. Flume PA, Robinson KA, O'Sullivan BP, et al.: Cystic fibrosis pulmonary guidelines: airway clearance therapies. Respir Care. 2009, 54:522-37.

3. Carman B, Cahill T, Warden G, McCall J: A prospective, randomized comparison of the Volume Diffusive Respirator vs conventional ventilation for ventilation of burned children. 2001 ABA paper. J Burn Care Rehabil. 2002, 23:444-8.

4. Reper P, Van Bos R, Van Loey K, et al.: High frequency percussive ventilation in burn patients: hemodynamics and gas exchange. Burns. 2003, 29:603-8. 10.1016/s0305-4179(03)00068-8

5. Cioffi WG Jr, Rue LW 3rd, Graves TA, McManus WF, Mason AD Jr, Pruitt BA Jr: Prophylactic use of highfrequency percussive ventilation in patients with inhalation injury. Ann Surg. 1991, 213:575-80; discussion 580-2. 10.1097/00000658-199106000-00007

6. Dmello D, Nayak RP, Matuschak GM: High-frequency percussive ventilation for airway clearance in cystic fibrosis: a brief report. Lung. 2010, 188:511-3. 10.1007/s00408-010-9252-5

7. Lucangelo U, Fontanesi L, Antonaglia V, et al.: High frequency percussive ventilation (HFPV). Principles and technique. Minerva Anestesiol. 2003, 69:841-8, 848-51.

8. Krishnan JA, Brower RG: High-frequency ventilation for acute lung injury and ARDS . Chest. 2000, 118:795807. 10.1378/chest.118.3.795

9. Freitag L, Long WM, Kim CS, Wanner A: Removal of excessive bronchial secretions by asymmetric highfrequency oscillations. J Appl Physiol (1985). 1989, 67:614-9. 10.1152/jappl.1989.67.2.614 